\title{
Transmit Beamforming with Reduced Feedback Information in OFDM Based Wireless Systems
}

\author{
Seung-Hyeon Yang, Jae-Yun Ko, and Yong-Hwan Lee \\ School of Electrical Engineering and INMC, Seoul National University \\ Kwanak P. O. Box 34, Seoul 151-600 Korea \\ Email: lis7@ttl.snu.ac.kr, paul@fruit.snu.ac.kr, ylee@snu.ac.kr
}

\begin{abstract}
This paper considers a transmit beamforming scheme that works with reduced feedback information in OFDM based multiple-input single-output (MISO) wireless systems. The proposed scheme generates the beamforming weight using information on the previous beamforming weights and channel correlation, significantly reducing the amount of feedback signaling burden. The feedback signaling overhead can further be reduced with the use of clustering and interpolation techniques. Simulation results show that the proposed scheme outperforms conventional beamforming techniques, while using the same amount of feedback signaling overhead.
\end{abstract}

\section{INTRODUCTION}

Multiple-input multiple-output (MIMO) systems can considerably improve the performance of wireless communication systems. They can easily be applied to orthogonal frequency division multiplexing (OFDM) schemes. The performance improvement due to the use of multiple antennas is deeply associated with the accuracy of channel state information (CSI). When the CSI is available at the transmitter, the system performance can significantly be improved by employing a transmit beamforming technique that can provide a diversity gain as well as array gain [1]-[3]. Optimum beamforming techniques, however, require accurate CSI at the transmitter, which may cause heavy feedback signaling overhead.

To employ beamforming techniques in MIMO-OFDM systems, the transmitter may require the CSI of each subcarrier, increasing the amount of feedback signaling overhead in linear proportion to the number of subcarriers. This problem can be elevated by using codebook based quantization methods, where the receiver determines the optimum beamforming vector from a finite set of vectors, called codebook, and sends the index of the corresponding vector to the transmitter using a feedback channel in the uplink [4]-[6]. The overhead can further be reduced by exploiting the frequency correlation [7], [8]. The size of the codebook can be reduced by recursively encoding the codeword with the use of previous ones [7]. The amount of feedback signaling information can further be reduced by employing a so-called clustering technique that combines adjacent subcarriers into a cluster and uses a same beamforming vector for these subcarriers [8]. Performance degradation due to the use of a large cluster size can be alleviated by interpolating the beamforming vectors in the frequency domain [8]. However, it may require additional phase information.

In this paper, we consider the reduction of the feedback signaling burden for the transmit beamforming by exploiting the channel correlation in the frequency domain. The beamforming vector can be generated in a sequential manner using the correlation characteristics between the adjacent subcarriers. When adjacent subcarriers are correlated to each other, the beamforming vector of a subcarrier can be represented in terms of that of its adjacent subcarriers and a vector corresponding to the difference between the two adjacent subcarriers. Since the vector corresponding to the difference will have a span smaller than the original one, the resulting quantization error will be smaller than that of the original one when the same amount of feedback signaling is considered. Further reduction can be achieved by using interpolation techniques with the use of a phase-dependent codebook.

This paper is organized as follows. Section II describes the system model in consideration. The proposed feedback reduction scheme is presented in Section III and the performance is verified by computer simulation in Section IV. Finally, Section V concludes the paper.

Notation: Bold upper and lower letters denote matrices and vectors, respectively. $(\cdot)^{T}$ and $(\cdot)^{*}$ denote the transpose and the conjugate transpose, respectively. $E\{\cdot\}$ denotes the expectation operator. $U(N, m)$ denotes a set of $N(m \times 1)$ dimensional vectors with unit norm. $\lceil x\rceil$ denotes the smallest integer larger than or equal to $x$.

\section{SYSTEM MODEL}

Consider an OFDM based wireless system with $n_{t}$ transmit antennas and a single receive antenna (i.e., multiple-input single-output (MISO) system) as illustrated in Fig. 1. We assume that the system utilizes $N_{c}$ subcarriers where the index of each subcarrier is denoted by $k$. When a signal $s(k)$ is transmitted using an $\left(n_{t} \times 1\right)$-dimensional beamforming weight vector $\mathbf{w}(k)$, the received signal can be represented as

$$
r(k)=\mathbf{h}(k) \mathbf{w}(k) s(k)+n(k), \quad k=0, \ldots, N_{c}-1
$$

where $\mathbf{h}(k)$ is the $\left(1 \times n_{t}\right)$-dimensional channel vector whose entities are independent and identically distributed (i.i.d.) complex Gaussian with zero mean and unit variance, and $n(k)$ 


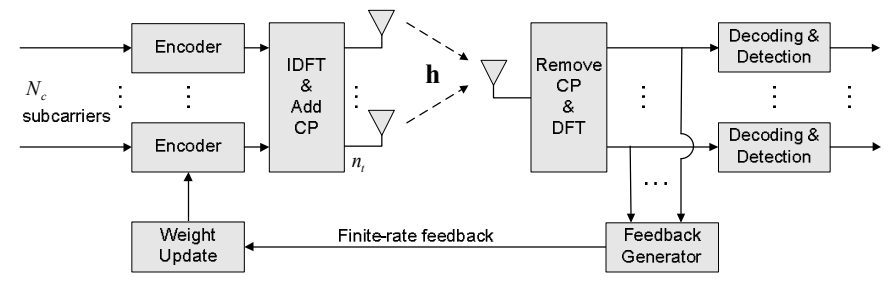

Fig. 1. Block diagram of an OFDM based MISO system with transmit beamforming.

is additive white Gaussian noise (AWGN). We assume that the CSI is perfectly estimated at the receiver and sent to the transmitter through a limited feedback channel without delay and error.

\section{Proposed Transmit Beamforming Scheme}

Assuming that the channel has correlation in the frequency domain, the channel vector of subcarrier $k$ can be expressed in terms of the channel vector of subcarrier $(k-1)$ as

$$
\mathbf{h}(k)=\rho \mathbf{h}(k-1)+\sqrt{1-|\rho|^{2}} \mathbf{e}
$$

where the correlation coefficient $\rho$ is defined by

$$
\rho=E\left\{\frac{\mathbf{h}(k) \mathbf{h}^{*}(k-1)}{\sqrt{\|\mathbf{h}(k)\|^{2}\|\mathbf{h}(k-1)\|^{2}}}\right\}
$$

and $\mathbf{e}$ is an $\left(1 \times n_{t}\right)$-dimensional vector whose entries are i.i.d. complex Gaussian with zero mean and unit variance. Note that the entries of $\mathbf{e}$ are independent of those of $\mathbf{h}(k-1)$.

Since the optimum beamforming vector can be determined by [6]

$$
\mathbf{w}(k)=\frac{\mathbf{h}^{*}(k)}{\|\mathbf{h}(k)\|},
$$

(2) can be rewritten as

$$
\mathbf{w}(k)=\rho^{*} \frac{\|\mathbf{h}(k-1)\|}{\|\mathbf{h}(k)\|} \mathbf{w}(k-1)+\sqrt{1-|\rho|^{2}} \frac{\|\mathbf{e}\|}{\|\mathbf{h}(k)\|} \mathbf{Z}
$$

where $\mathbf{z}=\mathbf{e}^{*} /\|\mathbf{e}\|$.

Unless the number of transmit antennas is too small, it can be shown that $\|\mathbf{h}(k-1)\| /\|\mathbf{h}(k)\| \approx\|\mathbf{e}\| /\|\mathbf{h}(k)\| \approx 1 \quad$ (refer to Appendix B) ${ }^{1}$. Then, (5) can further be simplified to

$$
\mathbf{w}(k) \approx \rho^{*} \mathbf{w}(k-1)+\sqrt{1-|\rho|^{2}} \mathbf{z} .
$$

Note that for a given beamforming vector $\mathbf{w}(k-1)$, the

\footnotetext{
${ }^{1}$ Simulation results show this assumption is quite valid if the number of transmit antennas is larger than 3 .
}

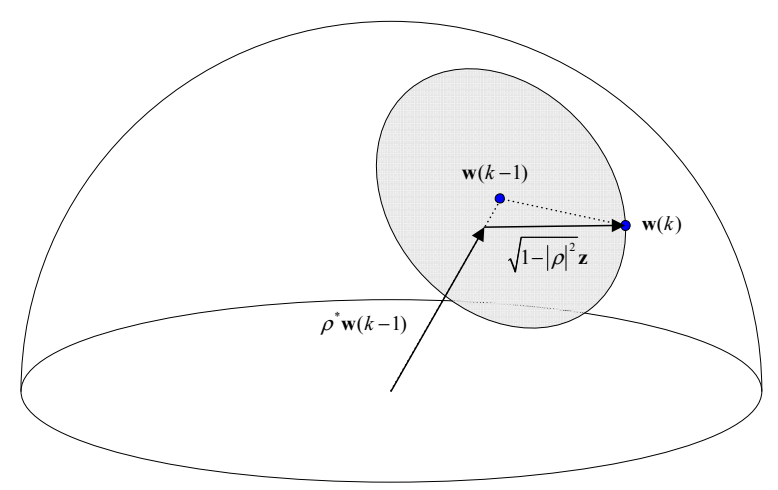

Fig. 2. Representation of $\mathbf{w}(k)$ by $\mathbf{w}(k-1)$.

beamforming vector for subcarrier $k$ can be represented in terms of the correlation coefficient $\rho$ and random vector $\mathbf{z}$. Since $\sqrt{1-|\rho|^{2}} \leq 1$, the effect of quantization error for $\mathbf{z}$ becomes smaller, improving the accuracy with the use of the same amount of feedback signaling for the beamforming weight. Moreover, since the correlation coefficient $\rho$ is not fast varying, the amount of additional feedback signaling overthed for the correlation coefficient may be marginal.

The random vector $\hat{\mathbf{z}}$ is quantized as

$$
\hat{\mathbf{z}}=\arg \max _{\mathbf{z} \in W} \mid \mathbf{h}(k)\left(\frac{\rho^{*} \mathbf{w}(k-1)+\sqrt{1-|\rho|^{2}} \mathbf{z}}{\left\|\rho^{*} \mathbf{w}(k-1)+\sqrt{1-|\rho|^{2}} \mathbf{z}\right\|} \mid\right.
$$

where $\boldsymbol{W}$ is the codebook whose $N$ entries are $\left(n_{t} \times 1\right)$ dimensional unit norm vectors. It can be seen that the beamforming vector is normalized to unit norm. The receiver feeds back the $\left\lceil\log _{2} N\right\rceil$-bit index of $\hat{\mathbf{z}}$ to the transmitter. After receiving the index, the transmitter recovers the beamforing vector for subcarrier $k$ as

$$
\mathbf{w}(k)=\frac{\rho^{*} \mathbf{w}(k-1)+\sqrt{1-|\rho|^{2}} \hat{\mathbf{z}}}{\left\|\rho^{*} \mathbf{w}(k-1)+\sqrt{1-|\rho|^{2}} \hat{\mathbf{z}}\right\|} .
$$

The codebook can be generated as [6], [9]

$$
\boldsymbol{W}=\arg \max _{\boldsymbol{X} \in U\left(N, n_{t}\right)} \delta(X) .
$$

Here, $\delta(\boldsymbol{X})$ is defined as

$$
\delta(\boldsymbol{X})=\min _{1 \leq i \leq j \leq N}\left\|\mathbf{x}_{i}-\mathbf{x}_{j}\right\|
$$

where $\mathbf{x}_{n}$ is $n$-th entry of $\boldsymbol{X}$. Note that in (9), the codebook is generated by maximizing the minimum Euclidean distance between any pair of two vectors in the codebook unlike conventional codebooks such as Grassmannian codebook [6]. 
TABLE I

SIMULATION CONDITION

\begin{tabular}{|c|c|}
\hline Number of subcarriers $\left(N_{c}\right)$ & 64 \\
\hline Number of transmit antennas $\left(n_{t}\right)$ & 4 \\
\hline Number of receive antennas $\left(n_{r}\right)$ & 1 \\
\hline Cluster size $(K)$ & 8 \\
\hline RMS delay spread & $167 \mathrm{~ns}$ \\
\hline Link adaptation & $\begin{array}{c}\text { Ideal (i.e., using the Shannon's } \\
\text { capacity curve) }\end{array}$ \\
\hline
\end{tabular}

The performance can further be improved by employing a frequency interpolation technique. Assume that $K$ subcarriers are combined into a cluster. The receiver can generate a beamforming weight corresponding to the representative subcarrrier for each cluster (e.g., the center subcarrier in each cluster). Then the beamforming weight of other subcarriers can be estimated from those of the representative subcarriers. For example, assuming that the beamforming vector for subcarrier $m K$, where $m=1,2, \ldots, N_{c} / K-1$, is reported from the receiver, the beamforming vector of other subcarriers can be estimated by linear interpolation as

$$
\hat{\mathbf{w}}(m K+l)=\frac{\left(1-\frac{l}{K}\right) \mathbf{w}(m K)+\frac{l}{K} \mathbf{w}(m K+K)}{\left\|\left(1-\frac{l}{K}\right) \mathbf{w}(m K)+\frac{l}{K} \mathbf{w}(m K+K)\right\|}
$$

where $m$ is the cluster index and $0 \leq l \leq K-1$. Note that the proposed scheme does not require any additional information (e.g., the phase information in [8]).

We consider the use of two different codebooks known at both the transmitter and the receiver; one for the initialization and the othe for the random vector. The proposed algorithm can be realized as follows:

1. Initialization: Select an initial state codebook for center subcarrier of the first cluster. The beamforming vector corresponding to the initial subcarrier is quantized into a $b_{1}$-bit codeword using a codebook comprising $N_{1}$ vectors generated by a conventional codebook method.

2. Update of the beamforming vector corrseponding to the center subcarrier of each cluster: After the initialization, the beamforming vector corrseponding to the center subcarrier of each cluster is calculated according to (7). The codebook comprising $N_{2}$ vectors is used to quantize the random vector $\mathbf{z}$ into a $b_{2}$-bit codeword.

3. Estimation of the beamforming vector for the rest of subcarriers: The transmitter calculates the beamforing vector corresponding to the center subcarrier of each cluster by (8) and then the beamforming vectors of other subcarriers by an interpolation method (e.g., (11)).

\section{PERFormanCE EVAluation}

The performance of the proposed scheme is verified by

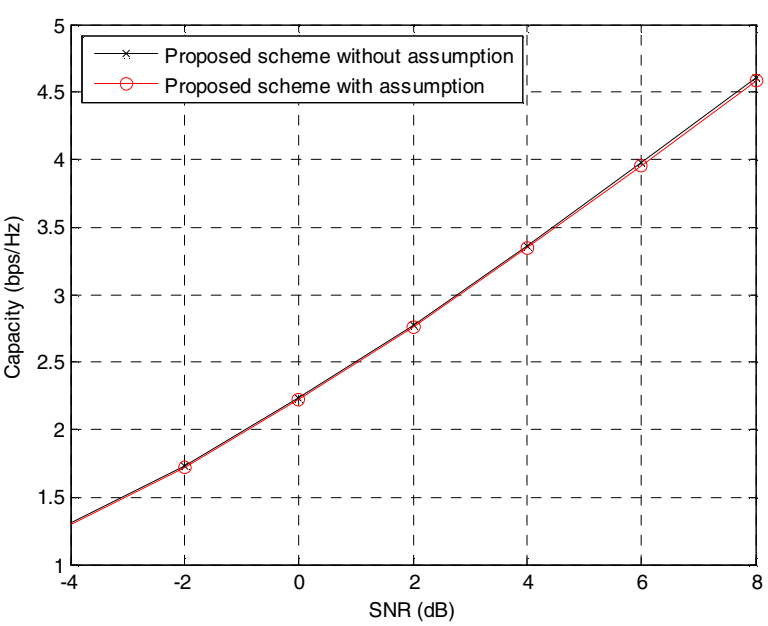

Fig. 3. Capacity with and without the assumption in (5) and (6).

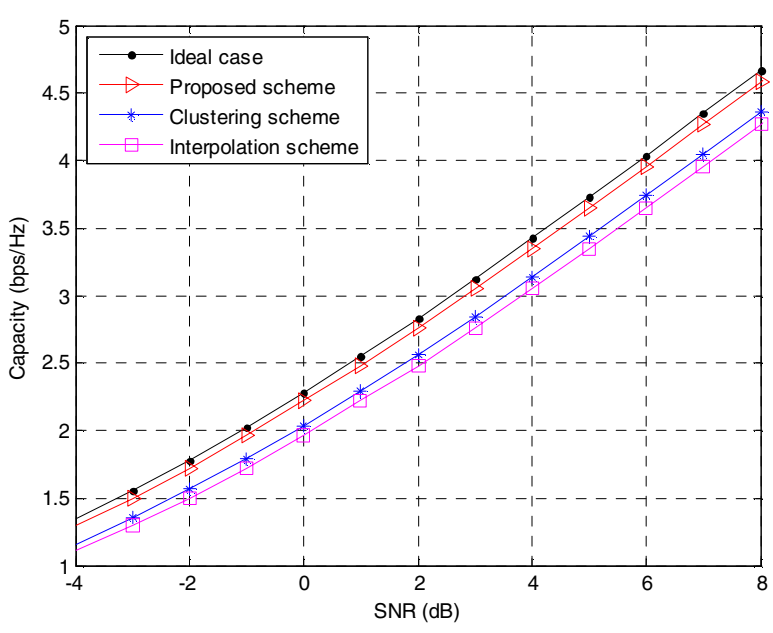

Fig. 4. Capacity achieved by the proposed scheme.

computer simulation. The simulation condition is summarized in Table I. The codebook can be found in Appendix A.

Fig. 3 compares the capacity of the proposed scheme with and without the assumption (5) and (6). The proposed scheme without the assumption means that the transmitter uses all the quantized random vector, norm value of the channel and norm value of the random vector by (5), whereas the one with the assumption means that the transmitter only uses the quantized random vector by (6). It can be seen that performance degradation due to the assumption is neglegible.

Fig. 4 depicts the performance of the proposed scheme in terms of the capacity when $b_{1}$ and $b_{2}$ are quantized into 6 bits, respectively. For comparison, we consider two conventional schemes, clustering and interpolation-based scheme in [7] and [8]. The clustering scheme uses the beamforming vector of the center subcarrier as the representative vector of the cluster and the interpolation-based 


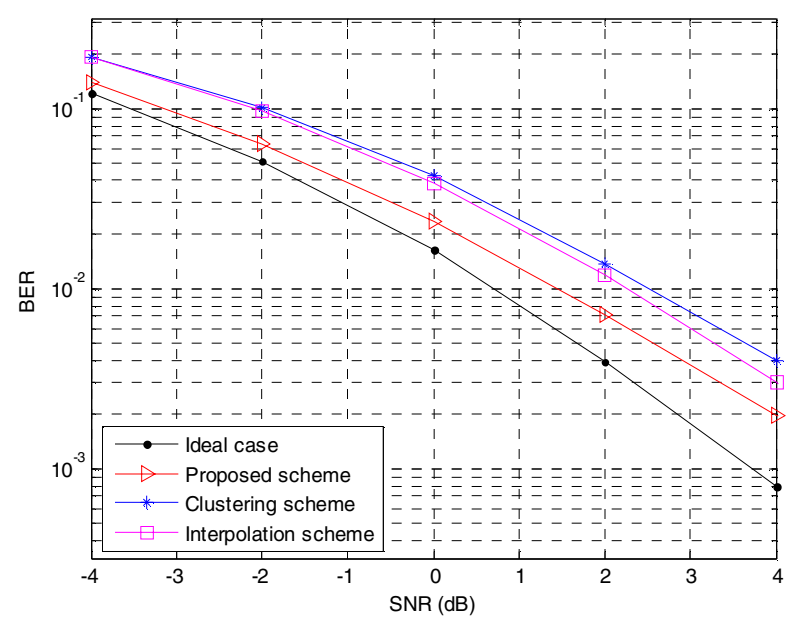

Fig. 5. BER performance of beamforming techniques.

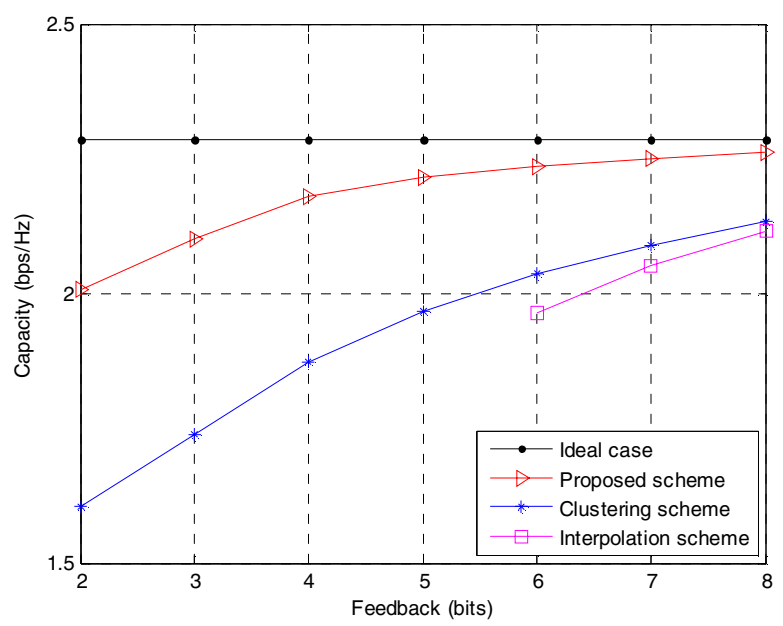

Fig. 6. Capacity comparison of beamforming techniques.

scheme additionally uses phase rotation information to interpolate the beamforming vectors. We assume that the clustering scheme uses 6 bits for the codebook and the interpolation-based scheme uses 4 bits for the codebook and 2 bits for the phase information. For a reference, we also consider the ideal case corresponding to the case when the transmitter has perfect channel information of all subcarriers with full precision. It can be seen that the proposed scheme outperforms conventional schemes and that the interpolationbased scheme has the worst performance due to the use of a reduced size codebook for additional phase information.

Fig. 5 compares the BER peformance of the proposed scheme with that of the conventional schemes when QPSK modulation is employed with a $1 / 2$-rate convolutional channel code. The interpolation-based scheme provides better BER performance than the clustering scheme since it can achieve a
TABLE A-1

CODEBOOK FOR $n_{t}=2$ AND $N=8$ ( 3 BITS)

\begin{tabular}{|c|c|}
\hline $0.0652+0.9153 \mathrm{i}$ & $-0.0420-0.9265 \mathrm{i}$ \\
$0.3972+0.0111 \mathrm{i}$ & $-0.3740-0.0080 \mathrm{i}$ \\
\hline$-0.6094-0.2378 \mathrm{i}$ & $0.7913+0.1887 \mathrm{i}$ \\
$0.6609-0.3680 \mathrm{i}$ & $-0.5617+0.1508 \mathrm{i}$ \\
\hline
\end{tabular}

TABLE A-2

\begin{tabular}{|c|c|c|c|}
\multicolumn{4}{c}{ CODEBOOK FOR $n_{t}=4$ AND $N=8$ ( 3 BITS $)$} \\
\hline $0.4410+0.4949 \mathrm{i}$ & $-0.3811-0.4570 \mathrm{i}$ & $-0.5939-0.2921 \mathrm{i}$ & $0.1026+0.3811 \mathrm{i}$ \\
$0.2388+0.3964 \mathrm{i}$ & $-0.2329-0.3407 \mathrm{i}$ & $0.0448+0.1096 \mathrm{i}$ & $-0.4236-0.7272 \mathrm{i}$ \\
$0.3304+0.0946 \mathrm{i}$ & $-0.4601-0.1094 \mathrm{i}$ & $0.6815+0.0755 \mathrm{i}$ & $0.2122+0.2507 \mathrm{i}$ \\
$0.4252+0.2178 \mathrm{i}$ & $-0.4658-0.1870 \mathrm{i}$ & $0.2108-0.1828 \mathrm{i}$ & $0.1267-0.1098 \mathrm{i}$ \\
\hline $0.4026-0.4749 \mathrm{i}$ & $0.0918-0.0625 \mathrm{i}$ & $0.3335-0.0962 \mathrm{i}$ & $-0.4195+0.6468 \mathrm{i}$ \\
$0.6404-0.4106 \mathrm{i}$ & $-0.5972+0.1163 \mathrm{i}$ & $-0.1500+0.2909 \mathrm{i}$ & $0.0896+0.1464 \mathrm{i}$ \\
$0.1380+0.0638 \mathrm{i}$ & $0.2509-0.6091 \mathrm{i}$ & $-0.1215+0.0657 \mathrm{i}$ & $0.0657+0.1015 \mathrm{i}$ \\
$-0.0362+0.0967 \mathrm{i}$ & $-0.0617+0.4239 \mathrm{i}$ & $0.1129-0.8606 \mathrm{i}$ & $-0.5917+0.1076 \mathrm{i}$ \\
\hline
\end{tabular}

diversity gain by using additional phase information [8]. It can be seen that the proposed scheme noticeably outperforms the conventional schemes.

Fig. 6 depicts the capacity of the proposed scheme associated with the amount of the feedback signaling overhead, which represents the amount of the bit size for the beam weight after the initialization. The proposed scheme uses $b_{1}=8$ bits codewords for the initialization. The interpolation scheme uses 2 bits for the phase information (i.e., it uses $\left(b_{2}-2\right)$ bits for the beam weight). It can be seen that the proposed scheme outperforms other schemes with the use of the same bit size. This impies that the proposed scheme needs less amount of feedback to provide the same performance, compared to other conventional schemes.

\section{CONCLUSIONS}

In this paper, we have proposed a new transmit beamforming scheme in OFDM based wireless MISO systems. The proposed scheme can reduce the amount of feedback signaling burden for the beam weight by generating the beamforming vector using information on the previous beamforming vector, channel correlation and quantized random vector. The simulation results show that the proposed scheme can estimate the channel information more accurately than conventional schemes using the same amount of feedback signaling burden, improving the system performance. This implies that the proposed scheme can reduce the amount of feedback signaling burden to provide the same performance as conventional schemes.

\section{APPENDIX}

\section{A. EXAMPLES OF CODEBOOKS}

Some examples of codebooks are given in Table A-1 and Table A-2. 


\section{B. Proof OF THE Norm CONVERGENCE}

Consider two column vectors, $\quad \mathbf{a}=\left[a_{1}, \ldots, a_{n_{t}}\right]^{T}$ and $\mathbf{b}=\left[b_{1}, \ldots, b_{n_{t}}\right]^{T}$, whose entries are zero-mean complex Gaussian random variables with unit variance. Letting $R_{1}=\|\mathbf{a}\|$, the probability density function (pdf) of $R_{1}$ can be expressed as [10]

$$
f_{R_{1}}\left(R_{1}\right)=\frac{2}{\Gamma\left(n_{t}\right)} R_{1}^{2 n_{t}-1} e^{-R_{1}^{2}}
$$

where $\Gamma(\cdot)$ denotes the Gamma function. The pdf of $R_{2}=\|\mathbf{b}\| \quad$ is the same as that of $R_{1}$. Letting $R=R_{1} / R_{2}=\|\mathbf{a}\| /\|\mathbf{b}\|$, it can be shown that the pdf of $R$ is

$$
\begin{aligned}
f_{R}(R) & =\int_{-\infty}^{\infty}|x| f_{R_{1}, R_{2}}(x R, x) d x \\
& =\int_{0}^{\infty} x f_{R_{1}}(x R) f_{R_{2}}(x) d x \\
& =\frac{4}{\left(\Gamma\left(n_{t}\right)\right)^{2}} R^{2 n_{t}-1} \int_{0}^{\infty} x^{4 n_{t}-1} e^{-\left(R^{2}+1\right) x^{2}} d x \\
& =2 R^{2 n_{t}-1}\left(1+R^{2}\right)^{-2 n_{t}} \frac{\Gamma\left(2 n_{t}\right)}{\left(\Gamma\left(n_{t}\right)\right)^{2}} .
\end{aligned}
$$

Thus, it can be seen that

$$
\lim _{n_{t} \rightarrow \infty} f_{R}(R)= \begin{cases}\infty, & \text { if } R=1, \\ 0, & \text { otherwise. }\end{cases}
$$

This implies that $R=\|\mathbf{a}\| /\|\mathbf{b}\| \approx 1$ as $n_{t}$ increases.

\section{REFERENCES}

[1] T. K. Y. Lo, "Maximum ratio transmission," IEEE Trans. Commun., vol. 47, no. 10, pp. 1458-1461, Oct. 1999.

[2] P. A. Dighe, R. K. Mallik and S. S. Jamuar, "Analysis of transmit-receive diversity in Rayleigh fading," IEEE Trans. Commun., vol. 51, no. 4, pp. 694-703, Apr. 2003.

[3] R. W. Heath Jr. and A. Paulraj, "A simple scheme for transmit diversity using partial channel feedback," in Proc. IEEE Asilomar Conf. Signals, Syst., Comput., vol. 2, pp. 1073-1078, Nov. 1998.

[4] D. J. Love and R. W. Heath Jr., "Equal gain transmission in multi-input multi-output wireless systems," IEEE Trans. Commun., vol. 51, no. 7, pp. 1102-1110, July 2003.

[5] K. K. Mukkavilli, A. Sabharwal, E. Erkip, and B. Aazhang, "On beamforming with finite rate feedback in multiple-antenna systems," IEEE Trans. Inf. Theory, vol. 49, no. 10, pp. 2562-2579, Oct. 2003.

[6] D. J. Love, R. W. Heath Jr. and T. Strohmer, "Grassmannian beamforming for multiple-input multiple-output wireless systems," IEEE Trans. Inf. Theory, vol. 49, no. 10, pp. 2735-2747, Oct. 2003.

[7] S. Zhou, B. Li and P. Willett, "Recursive and trellis-based feedback reduction for MIMO-OFDM with rate-limited feedback," IEEE Trans. Wireless Commun., vol. 5, no. 12, pp. 3400-3405, Dec. 2006.

[8] J. Choi and R. W. Heath, Jr., "Interpolation based transmit beamforming for MIMO-OFDM with limited feedback," in Proc. Int. Conf. Commun., vol. 1, pp. 249-253, June 2004.

[9] A. Gersho and R. M. Gray, Vector Quantization and Signal Compression, Kluwer Academic Publishers, 1992.

[10] B. H. Hochwald, L. Marzetta and V. Tarokh, "Multiple-antenna channel hardening and its implications for rate feedback and scheduling," IEEE Trans. Inf. Theory, vol. 50, no. 9, pp. 1893-1909, Sept. 2004.

[11] C. K. Au-Yeung and D. J. Love, "On the performance of random vector quantization limited feedback beamforming in a MISO system," IEEE Trans. Wireless Commun., vol. 6, no. 2, pp. 458-462, Feb. 2007. 